\title{
Front Matter: Volume 6568
}

, "Front Matter: Volume 6568," Proc. SPIE 6568, Algorithms for Synthetic Aperture Radar Imagery XIV, 656801 (16 May 2007); doi: 10.1117/12.741109

SPIE Event: Defense and Security Symposium, 2007, Orlando, Florida, United States 


\section{PROCEEDINGS OF SPIE}

\section{Algorithms for Synthetic Aperture Radar Imagery XIV}

Edmund G. Zelnio

Frederick D. Garber

Editors

10-11 April 2007

Orlando, Florida, USA

Sponsored and Published by

SPIE-The International Society for Optical Engineering

Volume 6568 
The papers included in this volume were part of the technical conference cited on the cover and title page. Papers were selected and subject to review by the editors and conference program committee. Some conference presentations may not be available for publication. The papers published in these proceedings reflect the work and thoughts of the authors and are published herein as submitted. The publisher is not responsible for the validity of the information or for any outcomes resulting from reliance thereon.

Please use the following format to cite material from this book:

Author(s), "Title of Paper," in Algorithms for Synthetic Aperture Radar Imagery XIV, edited by Edmund G. Zelnio, Frederick D. Garber, Proceedings of SPIE Vol. 6568 (SPIE, Bellingham, WA, 2007) Article CID Number.

ISSN 0277-786X

ISBN 9780819466907

Published by

SPIE-The International Society for Optical Engineering

P.O. Box 10, Bellingham, Washington 98227-0010 USA

Telephone 1 360/676-3290 (Pacific Time) · Fax 1 360/647-1445

http://www.spie.org

Copyright (C) 2007, The Society of Photo-Optical Instrumentation Engineers

Copying of material in this book for internal or personal use, or for the internal or personal use of specific clients, beyond the fair use provisions granted by the U.S. Copyright Law is authorized by SPIE subject to payment of copying fees. The Transactional Reporting Service base fee for this volume is $\$ 18.00$ per article (or portion thereof), which should be paid directly to the Copyright Clearance Center (CCC), 222 Rosewood Drive, Danvers, MA 01923. Payment may also be made electronically through CCC Online at http://www.copyright.com. Other copying for republication, resale, advertising or promotion, or any form of systematic or multiple reproduction of any material in this book is prohibited except with permission in writing from the publisher. The CCC fee code is 0277 $786 \times / 07 / \$ 18.00$

Printed in the United States of America. 


\section{Contents}

vii Conference Committee

SESSION 1 ADVANCED RADAR IMAGING

656802 GOTCHA experience report: three-dimensional SAR imaging with complete circular apertures [6568-01]

E. Ertin, C. D. Austin, S. Sharma, R. L. Moses, L. C. Potter, The Ohio State Univ. (USA)

656803 Three-dimensional point spread function characterization of a radially displaced scatterer [6568-02]

U. K. Majumder, M. A. Temple, Air Force Institute of Technology (USA); M. J. Minardi,

E. G. Zelnio, Air Force Research Lab. (USA)

656804 Three-dimensional resolution for circular synthetic aperture radar [6568-03]

L. J. Moore, Univ. of Dayton (USA); L. C. Potter, The Ohio State Univ. (USA)

656805 Exploitation of UAV trajectories with perturbation for intelligent circular SAR applications [6568-04]

A. K. Mitra, T. Lewis, L. Willemsen, Air Force Research Lab. (USA)

656806 Synthetic aperture hitchhiker imaging using non-cooperative sources of opportunity [6568-05]

C. E. Yarman, B. Yazıcı, M. Cheney, Rensselaer Polytechnic Institute (USA)

656807 Bistatic synthetic aperture inversion for arbitrary flight trajectories [6568-06]

C. E. Yarman, B. Yazıcı, M. Cheney, Rensselaer Polytechnic Institute (USA)

656809 Characterizing geolocation ambiguity responses in synthetic aperture radar: ground moving target indication [6568-35]

M. E. Holston, Air Force Institute of Technology (USA); M. J. Minardi, Air Force Research Lab. (USA); M. A. Temple, M. A. Saville, Air Force Institute of Technology (USA)

Pagination: Proceedings of SPIE follow an e-First publication model, with papers published first online and then in print and on CD-ROM. Papers are published as they are submitted and meet publication criteria. A unique, consistent, permanent citation identifier (CID) number is assigned to each article at the time of the first publication. Utilization of CIDs allows articles to be fully citable as soon they are published online, and connects the same identifier to all online, print, and electronic versions of the publication.

SPIE uses a six-digit CID article numbering system in which:

- The first four digits correspond to the SPIE volume number.

- The last two digits indicate publication order within the volume using a Base 36 numbering system employing both numerals and letters. These two-number sets start with $00,01,02,03,04,05,06,07,08,09,0 A, 0 B$... 0Z, followed by 10-1Z, 20-2Z, etc.

The CID number appears on each page of the manuscript. The complete citation is used on the first page, and an abbreviated version on subsequent pages. 
65680A A comparison of nonquadratic regularization implementations on the backhoe data set [6568-08]

A. S. Kondrath, B. D. Rigling, Wright State Univ. (USA)

65680B Joint enhancement of multichannel SAR data [6568-09]

N. Ramakrishnan, E. Ertin, R. L. Moses, The Ohio State Univ. (USA)

65680D A challenge problem for 2D/3D imaging of targets from a volumetric data set in an urban environment [6568-36]

C. H. Casteel, Jr., L. A. Gorham, M. J. Minardi, S. M. Scarborough, K. D. Naidu,

U. K. Majumder, Air Force Research Lab. (USA)

65680E Raider tracer: a MATLAB-based electromagnetic scattering simulator [6568-11]

B. D. Rigling, Wright State Univ. (USA)

65680F Indoor range for spatially resolved radar signature acquisition of targets [6568-12]

U. Aulenbacher, Ingenieurbüro für Sensorik und Signalverarbeitung (Germany); M.-A. John,

C. Inaebnit, armasuisse (Switzerland)

$65680 \mathrm{G}$ 3D characterization of radar targets by means of ISAR/SAR near field imaging techniques [6568-13]

M.-A. John, armasuisse (Switzerland); U. Aulenbacher, Ingenieurbüro für Sensorik und

Signalverarbeitung (Germany); C. Inaebnit, armasuisse (Switzerland)

65680H An end-to-end simulator for high-resolution spaceborne SAR systems [6568-14]

R. Speck, P. Turchi, H. Süß, German Aerospace Center (Germany)

65680I An algorithm for target validation using 3D scattering features [6568-15]

A. M. Raynal, The Univ. of Texas at Austin (USA); R. Bhalla, SAIC (USA); H. Ling, The Univ. of Texas at Austin (USA); V. J. Velten, Air Force Research Lab. (USA)

65680J Image exploitation for MISAR [6568-16]

N. Heinze, Fraunhofer Institut für Informations-und Datenverarbeitung (Germany);

M. Edrich, EADS Deutschland GmbH (Germany); G. Saur, W. Krüger, Fraunhofer Institut für Informations-und Datenverarbeitung (Germany)

65680K A comparison between imaging radar and medical imaging polar format algorithm implementations [6568-34]

L. A. Gorham, B. D. Rigling, E. G. Zelnio, Wright State Univ. (USA)

65680L SAR data exploitation: computational technology enabling SAR ATR algorithm development [6568-18]

U. K. Majumder, C. H. Casteel, P. Buxa, M. J. Minardi, E. G. Zelnio, Air Force Research Lab.

(USA); J. W. Nehrbass, Ohio Supercomputer Ctr. (USA)

\section{SESSION 3 DETECTION TECHNIQUES}

65680M An improved multi-channel clutter suppression algorithm [6568-19]

B. Kahler, B. Keaffaber, General Dynamics (USA) 
65680N Waveform preconditioning for clutter rejection in multipath for sparse distributed apertures [6568-20]

T. Varslot, B. YazıcI, C.-E. Yarman, M. Cheney, Rensselaer Polytechnic Institute (USA);

L. Scharf, Colorado State Univ. (USA)

656800 Multifrequency space time orthogonal projection (MF-STOP): a radar signal processing algorithm for detecting and discriminating targets in heavy clutter [6568-21]

Y. Tamrat, C. Hatleberg, Apogen Technologies (USA)

65680Q Motion-segmentation based change detection [6568-23]

B. Han, W. Roberts, D. WU, J. Li, Univ. of Florida (USA)

\section{SESSION 4 CLASSIFICATION TECHNIQUES}

65680R Identifiability of 3D attributed scattering features from sparse nonlinear apertures [6568-25] J. A. Jackson, R. L. Moses, Ohio State Univ. (USA)

65680S Improving ATR performance through distance metric learning [6568-26]

Y. Sun, M. Xue, J. Li, Univ. of Florida (USA); S. R. Stanfill, Lockheed Martin, Missiles and Fire Control (USA)

65680T Preprocessing of SAR interferometric data using anisotropic diffusion filter [6568-28] K. Sartor, J. De Vaughn Allen, E. Ganthier, Harris Corp. (USA); G. B. Tenali, Florida Institute of Technology (USA)

65680U Robust feature-based object tracking [6568-29]

B. Han, W. Roberts, D. WU, J. Li, Univ. of Florida (USA)

65680 Multinomial pattern matching for high range resolution radar profiles [6568-30]

M. L. Koudelka, J. A. Richards, M. W. Koch, Sandia National Labs. (USA)

65680X Diffusion maps and radar data analysis [6568-38]

Y. S. Bhat, Univ. of Minnesota (USA); G. Arnold, Air Force Research Lab. (USA)

65680Y Dissecting the object-image metric with image registration in mind [6568-32]

O. L. Mendoza, G. Arnold, Air Force Research Lab. (USA)

$65680 Z$ Score-based SAR ATR performance model with operating condition dependencies [6568-33]

V. I. Kaufman, BAE Systems AIT (USA); T. D. Ross, Air Force Research Lab. (USA); E. M. Lavely, BAE Systems AIT (USA); E. P. Blasch, Air Force Research Lab. (USA)

\section{POSTER SESSION}

656810 SAR images generated using the least mean squares (LMS) algorithm [6569-37]

J.E. Minardi II, Carroll High School (USA); M.J. Minardi, Air Force Research Lab. (USA)

Author Index 
Downloaded From: https://www.spiedigitallibrary.org/conference-proceedings-of-spie on 26 Apr 2023

Terms of Use: https://www.spiedigitallibrary.org/terms-of-use 


\title{
Conference Committee
}

\author{
Symposium Chair \\ John C. Carrano, Luminex Corporation (USA) \\ Symposium Cochair
}

Larry B. Stotts, Defense Advanced Research Projects Agency (USA)

Program Track Chair

Ivan Kadar, Interlink Systems Sciences, Inc. (USA)

Conference Chairs

Edmund G. Zelnio, Air Force Research Laboratory (USA) and Wright State University (USA)

Frederick D. Garber, Wright State University (USA)

Program Committee

Bir Bhanu, University of California, Riverside (USA)

Mujdat Çetin, Sabanci University (Turkey)

Dan E. Dudgeon, BAE Systems PIC. (USA)

Gil J. Ettinger, BAE Systems Advanced Information Technologies (USA)

Robert A. Hummel, Defense Advanced Research Projects Agency (USA)

Charles V. Jakowatz, Jr., Sandia National Laboratories (USA)

Eric R. Keydel, Science Applications International Corporation (USA)

John M. Miller, Army Research Laboratory (USA)

Randolph L. Moses, The Ohio State University (USA)

Brian D. Rigling, Wright State University (USA)

Timothy D. Ross, Air Force Research Laboratory (USA)

Gerard W. Titi, BAE Systems Advanced Information Technologies (USA)

Stephen P. Welby, Defense Advanced Research Projects Agency (USA)

Robert L. Williams, Air Force Research Laboratory (USA)

Session Chairs

$1 \quad$ Advanced Radar Imaging

Gerard W. Titi, BAE Systems Advanced Information Technologies (USA)

2 Radar Modeling and Measurement

Charles V. Jakowatz, Jr., Sandia National Laboratories (USA) 
3 Detection Techniques

Edmund G. Zelnio, Air Force Research Laboratory (USA) and Wright State University (USA)

4 Classification Techniques

Eric R. Keydel, Science Applications International Corporation (USA) 